\title{
Experimental evolution of Bacillus subtilis on Arabidopsis thaliana roots reveals fast adaptation and improved root colonization in the presence of soil microbes
}

Mathilde Nordgaard ${ }^{1}$, Christopher Blake ${ }^{1}$, Gergely Maróti ${ }^{2}$, Mikael Lenz Strube ${ }^{3}$, Ákos T. Kovács ${ }^{1}$,

${ }^{1}$ Bacterial Interactions and Evolution Group, DTU Bioengineering, Technical University of Denmark, 2800

Kongens Lyngby, Denmark

${ }^{2}$ Institute of Plant Biology, Biological Research Centre, Eötvös Loránd Research Network (ELKH), 6726

Szeged, Hungary

${ }^{3}$ Bacterial Ecophysiology and Biotechnology Group, DTU Bioengineering, Technical University of Denmark, 2800 Kongens Lyngby, Denmark

*For correspondence. E-mail: atkovacs@dtu.dk; Søltofts Plads Building 221, 2800 Kgs Lyngby, 12

Denmark, Tel. +45 4525 2527; Fax +45 45884922

Running title: Adaption of $B$. subtilis on plant roots

Keywords: Bacillus subtilis; plant-microbe interaction; experimental evolution; biofilm, motility, microbial community 


\section{Abstract}

The soil ubiquitous Bacillus subtilis is known to promote plant growth and protect plants against disease. These characteristics make $B$. subtilis highly relevant in an agricultural perspective, fueling the interest in studying B. subtilis-plant interactions. Here, we employ an experimental evolution approach to explore adaptation of $B$. subtilis to Arabidopsis thaliana roots. B. subtilis rapidly adapts to the plant root environment, as evidenced by improved root colonizers observed already after 12 consecutive transfers between seedlings in a hydroponic setup. Further phenotypic characterization of evolved isolates from transfer 30 revealed that increased root colonization was associated with robust biofilm formation in response to the plant polysaccharide xylan. Additionally, several evolved isolates across independent populations were impaired in motility, a redundant trait in the selective environment. Interestingly, two evolved isolates outcompeted the ancestor during competition on the root but suffered a fitness disadvantage in non-selective environment, demonstrating an evolutionary cost of adaptation to the plant root. Finally, increased root colonization by a selected evolved isolate was also demonstrated in the presence of resident soil microbes. Our findings provide novel insights into how a well-known PGPR rapidly adapts to an ecologically relevant environment and reveal evolutionary consequences that are fundamental to consider when evolving strains for biocontrol purposes. 


\section{Introduction}

The nutrient-rich rhizosphere is a hotspot for microbial activity, containing up to $10^{11}$ bacteria per gram root [1] and housing more than 30.000 prokaryotic species [2]. Among those are beneficial bacteria which are actively recruited by the plant through root exudate secretion and subsequently colonize the root from where they benefit the plant through various mechanisms [2-6]. One well-known plant growthpromoting rhizobacterium (PGPR) is the spore-forming Bacillus subtilis which has been isolated from various plant species [7-10]. Its plant-beneficial traits [11] and promising role as a biocontrol agent [1214] have fueled the interest in studying $B$. subtilis-plant interactions and led to the elucidation of mechanisms involved in the establishment on the root and behind its plant beneficial properties.

An obvious prerequisite for successful root colonization is the ability of the bacterium to reach the plant root. Chemotaxis towards root exudates was shown to be important for the early colonization of Arabidopsis thaliana roots by B. subtilis under hydroponic conditions [15], while solid surface motility was suggested to play a role during tomato root colonization in vermiculites [16]. After reaching the plant root, B. subtilis initiates biofilm $[15,17-19]$. Similar to in vitro conditions, plant-associated biofilms depend on the production of the matrix components EPS and TasA [18-21]. Expression of the operons involved in matrix production, epsA-O and tapA-sip $W$-tas $A$, is controlled by the biofilm repressor $\operatorname{SinR}[22,23]$. In response to environmental cues, one or more of the five histidine kinases, KinA-E, are activated resulting in phosphorylation of the master regulator Spo0A through a phosphorelay [24]. At threshold concentrations of SpoOA P, Sinl is produced [25], which binds to and inhibits SinR [26], resulting in matrix gene expression. As an environmental cue for root colonization, root exudate from tomato was shown to trigger biofilm formation in B. subtilis in a KinD-dependent manner [27], while another study reported biofilm induction by plant polysaccharides via KinC and KinD [19]. B. subtilis benefits from such microbeplant interactions by acquiring carbon source from the plants. In turn, $B$. subtilis protects the plant against disease directly by producing antimicrobials $[12,17,18,28]$, indirectly through niche competition $[29,30]$, 
and elicitation of induced systemic resistance in the plant $[4,31]$. Moreover, $B$. subtilis promotes plant growth by improving nutrient availability and producing growth-promoting phytohormones [11].

Mutualistic bacteria-plant interactions are a result of a long-term co-evolution of bacteria and plants that started by the colonization of land by ancestral plants 450 million years ago [32]. Here, we were interested in studying how $B$. subtilis adapts to plant roots on a much shorter evolutionary time scale. Experimental evolution (EE) provides a powerful tool to study microbial adaptation to different environments in realtime $[33,34]$. We recently studied EE of $B$. subtilis on $A$. thaliana plants roots, which revealed diversification of $B$. subtilis into three distinct morphotypes that interacted synergistically during root cocolonization [11]. Such morphological diversification has also been observed in EE of $B$. subtilis biofilm pellicles formed at the air-liquid interface [35] as well as during EE of Burkholderia cenocepacia biofilms on polystyrene beads [36]. In this study, we employed EE of $B$. subtilis on $A$. thaliana roots under axenic conditions to investigate which phenotypes are altered during adaptation to the plant root. We found that $B$. subtilis rapidly adapted to the root as observed by improved root colonizers already at transfer 12 . After 30 transfers, evolved isolates displayed robust biofilm formation in response to plant polysaccharides, impaired motility, and altered growth on plant compounds. We further discuss a few selected genetic changes possibly underlying the adaptation to the plant root. Finally, we demonstrate that adaptation of $B$. subtilis to $A$. thaliana roots is accompanied by an evolutionary cost, and report an evolved isolate displaying increased root colonization also in the presence of resident soil microbes. 


\section{Materials and methods}

\section{Bacterial strains and cultivation methods}

All strains used in this study are listed in Table 1. Bacillus subtilis DK1042 strain, an easily transformable derivative of the undomesticated $B$. subtilis NCBI 3610, was used as the ancestor for the experimental evolution [37]. For pairwise competitions between the ancestor and evolved isolates, and for cocolonization with a semi-synthetic, soil-derived community, the evolved isolates derived from the ancestor were transformed with plasmids pTB497.1 and pTB498.1. These plasmids harbor the gfp and mKATE gene, respectively, under the control of the hyper-spank (constitutive) promoter and a spectinomycin resistance gene within the flanking regions of the amyE gene. Transformants were identified by selection for spectinomycin resistance and double crossovers were verified by the loss of amylase activity. The four bacterial species, Pedobacter sp., Rhodococcus globerulus, Stenotrophomas indicatrix and Chryseobacterium sp., constituting a semi-synthetic, soil-derived community were acquired in Dyrehaven, Kongens Lyngby, Denmark ( $55^{\circ} 47^{\prime} 19.68^{\prime \prime} \mathrm{N}, 12^{\circ} 33^{\prime} 29.88^{\prime \prime} \mathrm{E}$ ), as described [38].

B. subtilis strains were routinely grown overnight $(\mathrm{ON})$ in Lysogeny Broth (LB; LB-Lennox, Carl Roth, Germany; $10 \mathrm{~g} / \mathrm{L}$ tryptone, $5 \mathrm{~g} / \mathrm{L}$ yeast extract and $5 \mathrm{~g} / \mathrm{L} \mathrm{NaCl}$ ) at $37{ }^{\circ} \mathrm{C}$ while shaking at $220 \mathrm{rpm}$. When relevant, spectinomycin was added at a final concentration of $100 \mu \mathrm{g} / \mathrm{mL}$. The bacterial species used for the four-member, soil-derived community were grown for $48 \mathrm{~h}$ in $0.1 \%$ (w/v) Tryptic Soy Broth (TSB; VWR, Radnor, PA, USA) at room temperature. For the experimental evolution on plants and for root colonization assays, a minimal salts nitrogen glycerol (MSNg) medium (5 mM potassium phosphate buffer [pH 7], 0.1 M Mops [pH 7], $2 \mathrm{mM} \mathrm{MgCl}, 50 \mu \mathrm{M} \mathrm{MnCl}_{2}, 1 \mu \mathrm{M} \mathrm{ZnCl}, 2 \mu \mathrm{M}$ thiamine, $700 \mu \mathrm{M} \mathrm{CaCl}_{2}, 0.2 \% \mathrm{NH}_{4} \mathrm{Cl}_{2}$ and $0.05 \%$ glycerol) was used. Pellicle biofilm formation was assessed in LB medium with or without a supplementation of xylan (0.5 \%) (w/v) (xylan from beechwood, Carl Roth, Germany). Pairwise 
competitions between the ancestor and evolved isolates were evaluated in LB + xylan ( $0.5 \%)$. Growth of

B. subtilis ancestor and evolved isolates alone or in co-culture with the semi-synthetic, soil-derived community was monitored in MSNc + xylan (5 mM potassium phosphate buffer [pH 7], $0.1 \mathrm{M}$ Mops [pH 7], $2 \mathrm{mM} \mathrm{MgCl}_{2}, 50 \mu \mathrm{M} \mathrm{MnCl} 2,1 \mu \mathrm{M} \mathrm{ZnCl}_{2}, 2 \mu \mathrm{M}$ thiamine, $700 \mu \mathrm{M} \mathrm{CaCl}_{2}, 0.2 \% \mathrm{NH}_{4} \mathrm{Cl}_{2}, 0.5 \%$ cellobiose, $0.5 \%$ xylan). Cellobiose and xylan were purchased from Carl Roth (Germany).

\section{Table 1: Strains used in this study}

\begin{tabular}{|c|c|c|}
\hline Name & Description & Reference \\
\hline DK1042 & B. subtilis NCIB 3610 com/Q121 & [37] \\
\hline TB500.1 & DK1042 amyE::P hyperspank -GFP Spec ${ }^{R}$ & \multirow[t]{4}{*}[21]{} \\
\hline TB501.1 & DK1042 amyE::P $P_{\text {hyperspank-mKATE Spec }}{ }^{R}$ & \\
\hline TB530.1 & DK1042 amyE::P hyperspank-GFP Spec $^{R}$ hag::Km ${ }^{R}$ & \\
\hline TB531.1 & 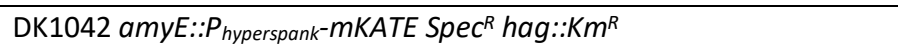 & \\
\hline DTUB310 & DK1042 Ev6.1 amyE::P hyperspank-GFP Spec $^{R}$ & \multirow{4}{*}{ This study } \\
\hline DTUB311 & DK1042 Ev6.1 amyE::P hyperspank- mKATE Spec ${ }^{R}$ & \\
\hline DTUB312 & DK1042 Ev7.3 amyE::Phyperspank-GFP Spec ${ }^{R}$ & \\
\hline DTUB313 & DK1042 Ev7.3 amyE::P hyperspank - mKATE Spec ${ }^{R}$ & \\
\hline D749 & Pedobacter sp & \multirow{4}{*}{ (Lozano-Andrade et al., 2021) } \\
\hline D757 & Rhodococcus globerulus & \\
\hline D763 & Stenotrophomas indicatrix & \\
\hline D764 & Chryseombacterium sp & \\
\hline MC1061 & 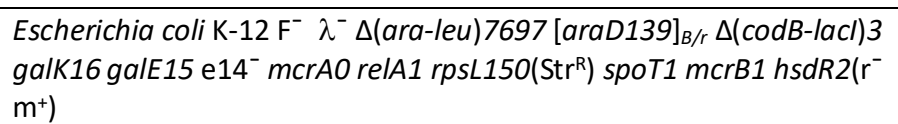 & [39] \\
\hline
\end{tabular}

\section{A. thaliana seedling preparation}

A. thaliana Col-0 seeds were surface sterilized in a $2 \%(\mathrm{v} / \mathrm{v})$ sodium hypochlorite solution and shaken on an orbital mixer for 12 minutes. The seeds were washed five times in sterile, distilled water. Approximately 
15 sterilized seeds were carefully pipetted onto pre-dried MS agar plates (Murashige and Skoog basal salts mixture, Sigma) (2.2 $\mathrm{g} \mathrm{l}^{-1}, \mathrm{pH}=5.6-5.8$ supplemented with $1 \%$ agar). Plates were sealed with parafilm and stratified at $4{ }^{\circ} \mathrm{C}$ for 3 days, and were then placed at an angle of $65^{\circ} \mathrm{C}$ in a plant chamber (cycles of $16 \mathrm{~h}$ light at $24^{\circ} \mathrm{C}$, and $8 \mathrm{~h}$ dark at $20^{\circ} \mathrm{C}$ ). The seedlings were grown in the plant chamber for $6-8$ days before use.

Experimental evolution of $B$. subtilis on $A$. thaliana seedlings

To study how $B$. subtilis adapts to $A$. thaliana roots, we employed an experimental evolution (EE) setup similar to the one established by Lin et al. (2021). This setup was inspired by a long-term EE on polystyrene beads [36], but instead of beads, $A$. thaliana roots were used for successive root colonization by $B$. subtilis. The EE on plant seedlings was carried out under axenic, hydroponic conditions optimized for B. subtilis $[19,40,41]$. Seven parallel populations were initiated by inoculating $A$. thaliana seedlings, placed in 300 $\mu \mathrm{L}$ MSNg medium in 48-well plates, with $B$. subtilis DK1042 at a starting $\mathrm{OD}_{600}$ of 0.02 . In addition, a replicate with a sterile root in MSNg without bacterial inoculation was included as a control. The 48-well plate was incubated in a plant chamber (cycles of $16 \mathrm{~h} \mathrm{light} \mathrm{at} 24{ }^{\circ} \mathrm{C} / 8 \mathrm{~h}$ dark at $20^{\circ} \mathrm{C}$ ) at mild agitation (90 rpm). After $48 \mathrm{~h}$, the colonized seedling was washed twice in MSNg and transferred to fresh medium containing a new, sterile seedling enabling re-colonization. Following this approach, the seven parallel populations were serially transferred every $48 \mathrm{~h}$ for a total of 32 days. At different time points during the ongoing EE, the old seedlings were washed twice in MSNg and vortexed with glass beads $(\varnothing 0.25-0.5 \mathrm{~mm}$, Carl Roth, Germany) to disperse the biofilm. The resulting cell suspension was used for plating to follow the productivity of the evolving populations, i.e. colony-forming unit (CFU) per root, and also preserved as frozen $\left(-80^{\circ} \mathrm{C}\right)$ stocks for later analysis. 
Isolation of single evolved isolates and colony morphology assay

Frozen stocks of population 3, 4, 6 and 7 from three different time points during the EE (transfer 12, 18 and 30) were streaked on LB plates to obtain single colonies of evolved isolates. Transfer 30 (T30) was designated as the final transfer for the isolation of evolved clones. From each population and time point, three colonies were randomly selected, prepared as ON cultures and saved as frozen stocks. Colony morphologies of evolved isolates were examined by spotting $2 \mu \mathrm{L}$ ON cultures on LB agar (1.5\%) and incubated at $30^{\circ} \mathrm{C}$ for $48 \mathrm{~h}$. Importantly, by growing the ancestor and evolved isolates from frozen stocks in LB ON, all isolates should be in a similar physiological state, so a potential difference in colony morphology should be attributed to genetic variation.

\section{Root colonization assay}

The evolved isolates were tested for root colonization under the same conditions applied during the EE. For individual root colonization, sterile $A$. thaliana seedlings in MSNg medium were inoculated with the ancestor or evolved isolates at a starting $\mathrm{OD}_{600}$ of 0.02 . For pairwise competition experiments, sterile $A$. thaliana seedlings were inoculated with a 1:1 mix of the ancestor and evolved isolates with opposite, fluorescent labels. Importantly, to obtain a similar starting cell number for the competition experiment, $\mathrm{ON}$ cultures of the ancestor and isolate Ev6.1 were adjusted to an $\mathrm{OD}_{600}$ of 0.2 , while Ev7.3 was adjusted to an $\mathrm{OD}_{600}$ of 1.0 corresponding to comparable total cell counts. For plant co-colonization by $B$. subtilis in the presence of a soil-derived synthetic community, cell suspensions of Pedobacter sp. D749 and Rhodococcus globerulus D757 were adjusted to an $\mathrm{OD}_{600}$ of 2.0, Stenotrophomas indicatrix D763 to an $\mathrm{OD}_{600}$ of 0.05 and Chryseobacterium sp. $\mathrm{D} 764$ to an $\mathrm{OD}_{600}$ of 0.1 and mixed by equal volumes (hereafter referred to as community). This mix was further adjusted to an $\mathrm{OD}_{600}$ of 0.2 . OD-adjusted $B$. subtilis ancestor $\left(\mathrm{OD}_{600}\right.$ of 0.2$)$ or Ev7.3 $\left(\mathrm{OD}_{600}\right.$ of 1.0$)$ and community were co-inoculated in four different ratios 
(0.1:1, 1:1, 10:1, 100:1 of $B$. subtilis and community, respectively) into MSNg medium containing a sterile A. thaliana seedling. The 48-well plates of all root colonization assays were incubated in the plant chamber at mild agitation $(90 \mathrm{rpm})$. After $48 \mathrm{~h}$, the colonized seedling was washed twice in MSNg and either vortexed with glass beads, and the resulting cell suspension plated for CFU quantification, or transferred to a glass slide for imaging using confocal laser scanning microscopy (CLSM).

For root colonization competition between B. subtilis WT and the $\Delta$ hag mutant, ON cultures of the WT and $\Delta$ hag mutant with opposite fluorescent labels were mixed 1:1 and inoculated onto sterile $A$. thaliana seedlings in MSNg medium at a starting $\mathrm{OD}_{600}$ of 0.02 . Plates were incubated in the plant chamber under static or shaking conditions ( $200 \mathrm{rpm}$ ) for $48 \mathrm{~h}$. The seedlings were then washed in MSNg and transferred to fresh medium containing a new, sterile seedling and incubated under the same conditions as before (i.e. static or shakeing conditions). This step was repeated once more, after which the third root was washed and vortexed with glass beads, and the resulting cell suspension was plated for CFU quantification.

Biofilm formation in response to plant polysaccharides

To test for pellicle biofilm formation in response to plant polysaccharides, the ancestor or the evolved isolates were inoculated into $1.5 \mathrm{~mL} \mathrm{LB}+$ xylan $(0.5 \%)$ medium in 24 -well plates to a starting $\mathrm{OD}_{600}$ of 0.05 and incubated under static conditions at $30^{\circ} \mathrm{C}$ for $48 \mathrm{~h}$.

Pairwise competition experiments in LB + xylan

ON cultures of the ancestor and selected evolved isolates were adjusted to an $\mathrm{OD}_{600}$ of 5.0. Ancestor was mixed 1:1 and 1:5 by volume with Ev6.1 and Ev7.3, respectively, to obtain a comparable number of starting cells. $80 \mu \mathrm{L}$ of the mix was inoculated into $20 \mathrm{~mL} \mathrm{LB}+$ xylan $(0.5 \%)$ medium in $100 \mathrm{~mL}$ bottles, 
giving a starting $\mathrm{OD}_{600}$ of 0.02 . Bottles were incubated at $37^{\circ} \mathrm{C}$ while shaking at $220 \mathrm{rpm}$ for $48 \mathrm{~h}$ followed by plating for CFU quantification.

Motility assays

Swimming motility was tested using soft agar plates (15 mL LB with $0.3 \%$ agar) dried for 5 minutes while swarming motility was evaluated on semi-soft agar plates (15 mL LB with $0.7 \%$ agar) dried for 20 minutes. $2 \mu \mathrm{L} \mathrm{ON}$ cultures of the ancestor or evolved isolates adjusted to an $\mathrm{OD}_{600}$ of 0.5 were spotted in the middle of a petri dish and incubated at $37^{\circ} \mathrm{C}$. Multiple stacking was avoided in order to keep a similar humidity across all plates. Swimming and swarming motility were followed for 6 and $8 \mathrm{~h}$, respectively. For each plate, motility was quantified as the averaged radius measured in four different directions.

Growth in the presence of xylan

To monitor the growth of ancestor and evolved isolates in the presence of plant compounds, two independent $\mathrm{ON}$ cultures of each isolate were independently inoculated into a 96-well plate containing $100 \mu \mathrm{L}$ MSNc + xylan $(0.5 \%)$ medium at a starting $\mathrm{OD}_{600}$ of $0.1 . \mathrm{OD}_{600}$ was monitored in a plate reader (BioTek Synergy HTX Multi-Mode Microplate Reader) every 15 min for $55 \mathrm{~h}$ at $24{ }^{\circ} \mathrm{C}$ under continuous shaking (orbital). To test for growth in co-culture with the community, two to three independent ON cultures of constitutively GFP-labelled B. subtilis ancestor and Ev7.3 were adjusted to an $\mathrm{OD}_{600}$ of 0.1 in MSNc + xylan (0.5\%). Cell suspensions of the four community members were adjusted in MSNc + xylan $(0.5 \%)$ to the same $\mathrm{OD}_{600}$ values used for the root colonization assay (see above), and the mixed community was adjusted to a final $\mathrm{OD}_{600}$ of 0.1 . Ancestor or Ev7.3 was co-inoculated with the community in the same ratios as for the root colonization assay $(0.1: 1,1: 1,10: 1$ and 100:1 of $B$. subtilis and 
community, respectively). $\mathrm{OD}_{600}$ and GFP were monitored in the plate reader every 15 minutes for $35 \mathrm{~h}$ at $24{ }^{\circ} \mathrm{C}$ under continuous shaking (orbital). In both growth assays, each well was measured at $9\left(\mathrm{OD}_{600}\right)$ or 5 (GFP $485 / 20 \mathrm{~nm})$ different points to avoid artifacts due to aggregation.

Pairwise interactions of $B$. subtilis with community members

To study potential altered interactions with any of the four bacterial species of the community, $2 \mu \mathrm{L}$ of ON cultures of $B$. subtilis ancestor or Ev7.3 adjusted to an $\mathrm{OD}_{600}$ of 0.5 was spotted on LB agar (1.5\%). On the same plate, $2 \mu \mathrm{L}$ of cell suspensions of Pedobacter sp. $\left(\mathrm{OD}_{600}\right.$ of 2.0$)$, Rhodococcus globerulus $\left(\mathrm{OD}_{600}\right.$ of 2.0), Stenotrophomas indicatrix $\left(\mathrm{OD}_{600}\right.$ of 0.1$)$ or Chryseobacterium sp. $\left(\mathrm{OD}_{600}\right.$ of 0.1$)$ were spotted at a $0.7 \mathrm{~cm}$ distance from the $B$. subtilis inoculum. The plates were incubated at $30^{\circ} \mathrm{C}$.

Microscopy/confocal laser scanning microscopy (CLSM)

Bright-field images of colonies, whole pellicle biofilms and pairwise interactions were acquired with an Axio Zoom V16 stereomicroscope (Carl Zeiss, Germany) equipped with a Zeiss CL 9000 LED light source and an AxioCam MRm monochrome camera (Carl Zeiss, Germany). Images of colonized seedlings were acquired using CLSM (Leica Microsystems Confocal Microscope SP8, Germany). The seedlings were washed twice in MSNg and placed onto glass slides. Images were obtained using the $63 \times / 1.4$ OIL objective. Fluorescent reporter excitation was performed with the argon laser at $488 \mathrm{~nm}$ while the emitted fluorescence of GFP and mKate was recorded at 484-536 nm and 567-654 nm, respectively. For each competition, three images from three independent seedlings were obtained. Representative images were used to visualize root colonization. Zen 3.1 Software (Carl Zeiss) and ImageJ was used for image visualization. 
Data and statistical analysis

Data and statistical analysis were carried out in Excel, Origin and R Studio. Outliers were identified using Dixon's test for outliers. For all statistical tests, normality was evaluated with a Shapiro-Wilk test. Equal variance was tested using F-test (for two groups) or Levene's test (for more than two groups). In addition, P-values were corrected for multiple testing using the Benjamini \& Hochberg (BH) procedure within each series of experiments. For all statistical tests, a significance level of 0.05 was used.

For individual root colonization, the log10-transformed productivity (CFU/mm root) of the replicates were divided by the mean of the log10-transformed productivity of the ancestor from the same experimental setup. For competition between WT and the $\Delta$ hag mutant, the observed frequency of the WT replicates after $48 \mathrm{~h}$ were divided by 0.5 (the starting frequency in the inoculation mix). For these experiments, the resulting normalized values were subjected to a One-sample $t$-test to test whether the mean was significantly different from 1 . For pairwise competitions between the ancestor and evolved isolates on the root or in LB + xylan, the relative fitness $(r)$ of the evolved isolate was calculated by comparing the frequency of the evolved isolate at the beginning and the end of the competition experiment as shown in equation 1 [42-44], in which $X_{0}$ is the initial and $X_{1}$ is the final frequency of the evolved isolate. The relative fitness was $\log 2$-transformed, and these values were subjected to a One-sample $t$-test to test whether the mean was significantly different from 0 .

1) Relative fitness $(r)=\frac{X_{1}\left(1-X_{0}\right)}{X_{0}\left(1-X_{1}\right)}$

For root co-colonization of the ancestor and Ev7.3 with the community, within each inoculation ratio, the difference between $B$. subtilis ancestor and Ev7.3, and between the communities co-inoculated with the ancestor and with Ev7.3, was tested with a Two-sample $t$-test. If the groups had unequal variance, Welch's Two-sample $t$-test was applied. 
For swimming and swarming motility, an ANOVA was performed on the log10-transformed data at each time point followed by a Dunnett's Multiple Comparison test using the ancestor as the control group.

For growth curve analysis of the ancestor and evolved isolates in monoculture, the carrying capacity (K) of individual replicates was calculated from the $\mathrm{OD}_{600}$ data using the Growthcurver-package in $\mathrm{R}$ [45]. Significant difference in carrying capacity between ancestor and evolved isolates was tested by an ANOVA followed by a Dunnett's Multiple Comparison test. Similarly, for the growth profiles of B. subtilis ancestor and Ev7.3 in co-culture with the community, the carrying capacity $(\mathrm{K})$ was calculated from the $\mathrm{GFP}_{485 / 20 \mathrm{~nm}}$ data. Significant difference between ancestor and Ev7.3 under the same inoculation ratio or alone was tested by a Two-sample $t$-test or Wilcoxon Unpaired Two-sample test (when data failed to meet parametric assumptions).

Genome re-sequencing and genome analysis

Resequencing of evolved isolates was performed as previously [35, 46-49]. Genomic DNA of selected evolved isolates from different time points during the EE was extracted from $2 \mathrm{~mL}$ ON cultures using the EURx Bacterial and Yeast Genomic DNA Kit. Paired-end libraries were prepared using the NEBNext ${ }^{\circledR}$ Ultra $^{\mathrm{TM}}$ II DNA Library Prep Kit for Illumina. Paired-end fragment reads were generated on an Illumina NextSeq sequencer using TG NextSeq ${ }^{\circledR}$ 500/550 High Output Kit v2 (300 cycles). Primary data analysis (base-calling) was carried out with "bcl2fastq" software (v2.17.1.14, Illumina). All further analysis steps were done in CLC Genomics Workbench. Reads were quality-trimmed using an error probability of $0.05(\mathrm{Q} 13)$ as the threshold. In addition, the first ten bases of each read were removed. Reads that displayed $\geq 80 \%$ similarity to the reference over $\geq 80 \%$ of their read lengths were used in the mapping. Non-specific reads were randomly placed to one of their possible genomic locations. Quality-based SNP and small In/Del variant calling was carried out requiring $\geq 8 \times$ read coverage with $\geq 25 \%$ variant frequency. Only variants supported by good quality bases $(Q \geq 20)$ were considered and only when they were supported by evidence from 
bioRxiv preprint doi: https://doi.org/10.1101/2021.07.09.451762; this version posted July 10, 2021. The copyright holder for this preprint (which

was not certified by peer review) is the author/funder, who has granted bioRxiv a license to display the preprint in perpetuity. It is made available under aCC-BY-NC-ND 4.0 International license.

both DNA strands in comparison to the B. subtilis NCIB 3610 genome and pBS plasmid (GenBank accession

no. NZ_CP020102 and NZ_CP020103, respectively). Identified mutations in each strain are listed in Supplementary Table 1. Raw sequencing data has been deposited to the NCBI Sequence Read Archive (SRA) database under BioProject accession number: PRJNA705352. 


\section{Results}

B. subtilis populations evolved on $A$. thaliana plant roots show rapid increase in root colonization

To explore how B. subtilis adapts to plant roots, we employed an experimental evolution (EE) setup previously established for another Bacillus species [50]. In short, B. subtilis DK1042 (ancestor) was inoculated onto $A$. thaliana seedlings under hydroponic conditions in seven parallel populations. The medium used ( $\mathrm{MSNg})$ is a minimal medium supplemented with a very low concentration of glycerol $(0.05$ $\%)$ and the bacteria thereby move towards the plant root to access carbon source. Every $48 \mathrm{~h}$, the colonized seedling was transferred to fresh medium containing a new sterile seedling, allowing selection for a regular cycle of dispersal from the root-associated biofilm, chemotaxis towards the new root and biofilm formation on the root surface. To follow potential changes in root colonization of the evolving populations during the ongoing $\mathrm{EE}$, the productivity, i.e. colony-forming unit (CFU) per root, was quantified at different time points. All seven independent populations showed a rapid increase in root colonization within the first seven transfers, after which the productivity of the populations remained rather stable with slight increases and drops dependent on the certain population (Fig. 1). While this rapid increase in productivity could be due to genetic adaptation to the root, the initial rise could also be caused by physiological adaptation to the experimental conditions. Interestingly, such a rapid increase in productivity of populations evolving on plant roots is consistent with our recent study where $B$. subtilis was evolved on older $A$. thaliana roots [11]. 


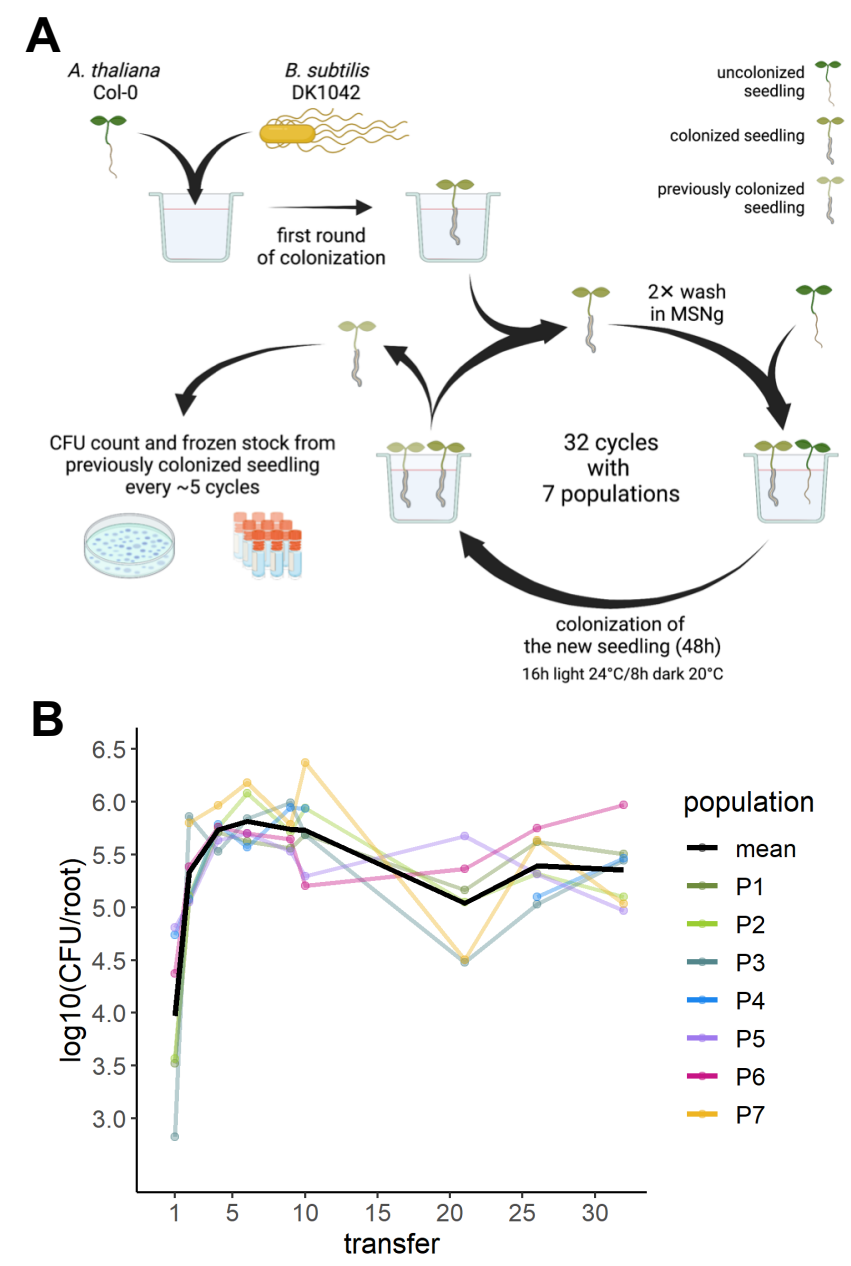

Fig. 1: Overview of experimental evolution and productivity of evolving populations. (A) Overview of the experimental evolution approach. (B) B. subtilis populations rapidly increase in productivity during experimental evolution on $A$. thaliana roots. The productivity (CFU/root) of the evolving populations was systematically quantified as CFU/root at 9 different time points during the ongoing EE. The $y$-axis displays the log10-transformed productivity. The black line represents the mean population productivity $(\mathrm{N}=7)$.

Several evolved isolates display altered colony morphologies

To examine whether genetic adaptation to the plant root had taken place during the EE and elucidate what phenotypes were altered after such plant root adaptation, single isolates from the evolved populations were saved as frozen stocks and subjected to phenotypic and genotypic characterization. To represent different populations and time points during the $E E$, three isolates were randomly picked from each of population 3, 4, 6 and 7 at transfer 12, 18 and 30 (hereafter referred to as T12, T18 and T30). To 
detect possible changes in colony morphology, ON cultures of the ancestor and evolved isolates were spotted on LB agar (1.5\%) and incubated at $30^{\circ} \mathrm{C}$ for $48 \mathrm{~h}$. On LB agar, the ancestor (DK1042) formed a round colony with a wrinkled periphery, while different colony morphologies were observed among the evolved isolates (Fig. 2). At T12, some isolates displayed a colony morphology resembling the one of the ancestor, e.g. isolate 3 from population $3(3.3)$ and isolate 2 from population 7 (7.2), referred to as the "Wrinkled"-type. Several other isolates formed a colony with a white sharp edge along the wrinkled periphery (including isolates 3.2, 4.3, 6.1 and 7.3), hereafter referred to as the "Sharp-Wrinkled"-type. Additionally, isolate 7.1 formed a hyper-wrinkled, white colony, referred to as the "Snow"-type. These distinct colony morphologies were also observed at later time points (T18 and T30). Interestingly, the Snow-type was only observed in population 7. Furthermore, the three isolates from population 6 at T30 formed slightly less wrinkled colonies compared to the ancestor. We note that three isolates do not represent the entire population, and isolates with other colony morphologies could be present in the populations. Nonetheless, the appearance of isolates with altered colony morphologies in the four populations indicates the presence of genetic changes. Furthermore, the occurrence of isolates with altered colony morphologies already at $\mathrm{T} 12$, and especially the presence of three different types (Wrinkled, Sharp-Wrinkled and Snow) in population 7, at this early time point, suggests rapid diversification of $B$. subtilis during EE on plant roots. Such diversification into distinct morphotypes was also observed in our previous study on EE of B. subtilis on plant roots [11] and has additionally been observed in EE of B. subtilis pellicle biofilms [35], indicating successful adaptation to the selective environment. 


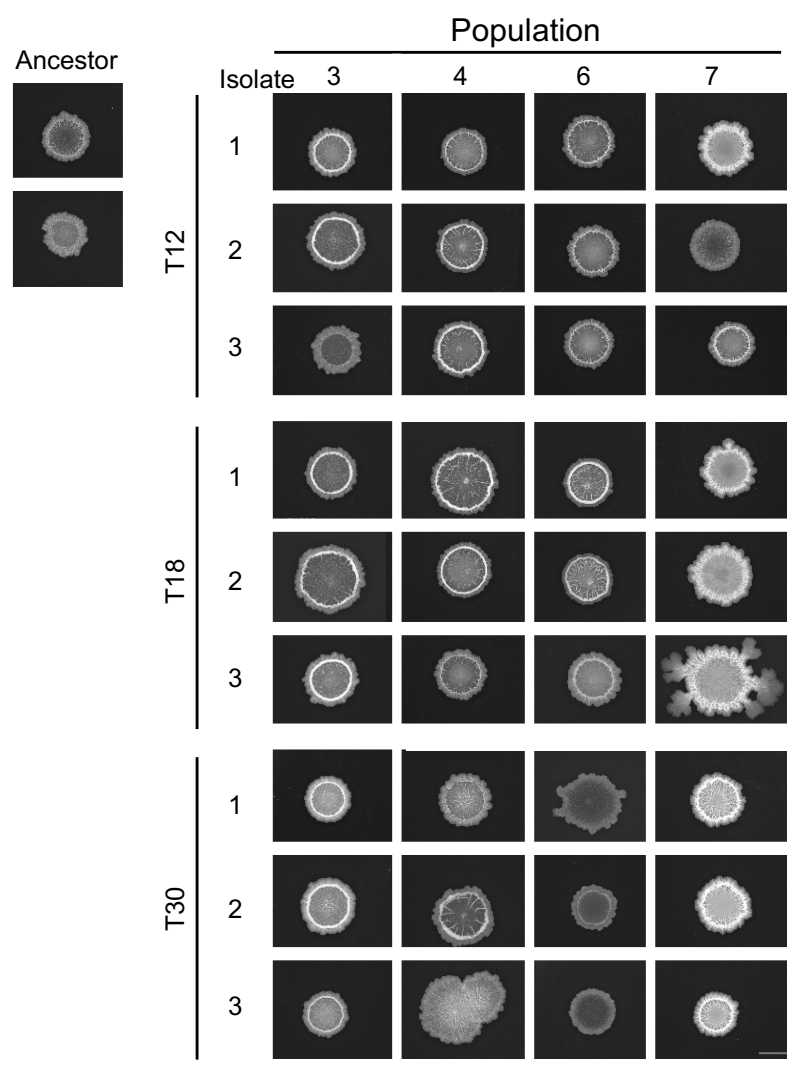

Fig. 2: Distinct colony morphologies are observed among evolved isolates from different time points of the experimental evolution. ON cultures of the ancestor and evolved isolates from populations 3, 4, 6 and 7 at transfer 12, 18 and 30 were spotted on LB agar (1.5\%) and imaged after incubation for $48 \mathrm{~h}$ at $30^{\circ} \mathrm{C}$ using a stereomicroscope. Ancestor represents $B$. subtilis DK1042. Each colony is representative of at least three replicates. Scale bar $=5 \mathrm{~mm}$.

Evolved isolates from different time points show increased colonization of $A$. thaliana roots

The design of the EE employed in this study should enable selection for bacteria that efficiently colonize the root. We, therefore, speculated whether the altered colony morphology of some of the evolved isolates was associated with improved productivity on the root (CFU/mm root). To test this, the ancestor or evolved isolates from the final time point (T30) were inoculated onto $A$. thaliana seedlings under the same conditions applied during the EE. CFU quantification revealed that most evolved isolates tended to show increased root colonization, with five isolates from three different populations displaying significantly increased productivity on the root, with an up to 1.3-fold change relative to the ancestor 
bioRxiv preprint doi: https://doi.org/10.1101/2021.07.09.451762; this version posted July 10, 2021. The copyright holder for this preprint (which was not certified by peer review) is the author/funder, who has granted bioRxiv a license to display the preprint in perpetuity. It is made available under aCC-BY-NC-ND 4.0 International license.

(Fig. 3). To track down when such improved root colonizers emerged during the EE, randomly selected evolved isolates from T12 and T18 were similarly tested. Three and five evolved isolates at T12 and T18, respectively, displayed significantly increased productivity relative to the ancestor. In addition, a single isolate from T12 was significantly reduced in root colonization. These results confirm that indeed genetic adaptation of $B$. subtilis to the plant root took place during the EE. Furthermore, the observation of improved root colonizers already at T12 suggests that the adaptation started rather early during the EE.

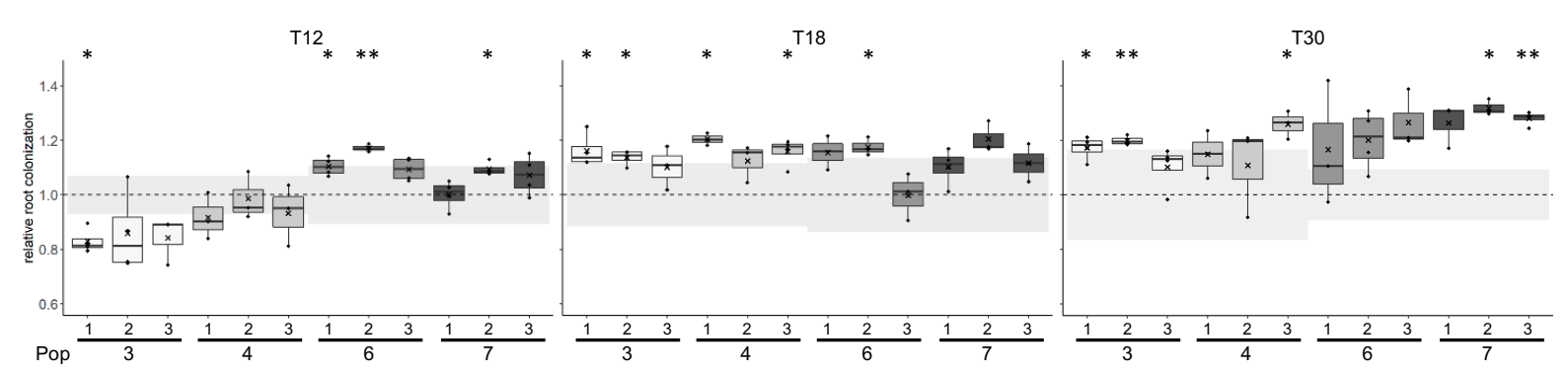

Fig. 3: Evolved isolates from different time points show increased productivity on the root relative to the ancestor. The ancestor and evolved isolates from populations $3,4,6$ and 7 at three different time points $(T 12,18,30)$ were tested for individual root colonization. For each evolved isolate, relative root colonization was calculated by dividing the log10-transformed productivity (CFU/mm root) of each replicate by the mean of the log10-transformed productivity of the ancestor from the same experimental setup. The dashed, horizontal line represents the mean of the ancestor, while the grey-shaded rectangles represent the standard deviation of the ancestor from the corresponding experiment. The normalized values were subjected to a One-sample $t$-test to test whether the mean was significantly different from 1 . P-values have been corrected for multiple comparisons. *: $\mathrm{P}<0.05,{ }^{* *}$ : $\mathrm{P}<0.01$.

Evolved isolates show altered pellicle biofilm formation in response to plant polysaccharides

To elucidate the phenotypes associated with adaptation to the plant root, evolved isolates from the final transfer (T30) were further characterized. For clarity the isolates at transfer 30 are named according to their population, i.e. isolate 1 from population 4 is referred to as "Ev4.1" and so forth. During root colonization, B. subtilis forms a biofilm on the root [17-19, 21]. To this end, plant polysaccharides (PPs) have been shown to induce biofilm formation in B. subtilis [19]. One way of adapting to the plant root is 
thereby through enhanced biofilm formation in response to PPs. To test whether the improved productivity on the root by the evolved isolates was associated with more robust biofilm formation in response to PPs, the ancestor and evolved isolates were tested for pellicle biofilm formation, a biofilm formed at the medium-air interface [51], in LB supplemented with xylan (LB + xylan). Xylan is a PP found among others in the secondary cell walls of $A$. thaliana [52], and one of the PPs that was shown to induce biofilm formation of $B$. subtilis when added into the generally non-biofilm inducing LB medium [19]. Importantly, a rich medium (LB) rather than the minimal medium (MSNg) was used in this assay to provide the bacteria with plenty of nutrients, allowing us to assess only the ability to form biofilm in response to xylan, and not the ability to utilize xylan for growth. A few isolates from T30 developed a pellicle similar to the ancestor, i.e. Ev4.1, Ev4.2 and Ev6.1 (Fig. 4A). In contrast, the remaining isolates developed more robust pellicles with highly structured wrinkles indicative of enhanced matrix production. Especially the three isolates from population 7 developed hyper-robust, white pellicles, consistent with the Snow-type colony morphology observed for these isolates (Fig. 2). The biofilms developed in response to xylan by the evolved isolates generally correlated with their productivity on the root. For example, isolates Ev4.1, Ev4.2 and Ev6.1 developing similar pellicles as the ancestor and isolates Ev7.1, Ev7.2 and Ev7.3 forming hyperwrinkled, robust pellicles in response to xylan were among the ones showing the smallest and largest increase in root colonization (Fig. 3 and 4A), respectively. This is in accordance with Chen et al. (2013) demonstrating that the ability of $B$. subtilis mutants to form robust biofilms in vitro correlated with that on the root. These results suggest that improved productivity on the root was associated with robust biofilm formation in response to xylan. To test whether this enhanced biofilm formation was specific to the presence of PPs, the ancestor and evolved isolates were tested for the ability to form pellicles in LB in the absence of xylan. In this medium, the pellicles developed by both the ancestor and evolved isolates were less robust (Fig. S1). For most isolates, the improved biofilm formation was specific to the presence 
of PPs, while the isolates from population 7 displayed robust biofilms also in the absence of plant compounds suggesting a general improvement in biofilm formation in these isolates.

Evolved isolates show reduced swarming and swimming motility

Another bacterial trait implicated in root colonization is motility. Chemotaxis and flagellar motility have been shown to be important for root colonization under hydroponic conditions [15]. We thereby speculated that motility could be under selection during the $\mathrm{EE}$, since enhanced motility would enable the bacteria to quickly reach the new root. To test this, the ancestor and evolved isolates from population 6 and 7 were tested for two types of motility: swimming motility, a single cell movement in aqueous environments powered by flagella rotation and swarming motility which is associated with a rapid multicellular movement of hyper-flagellated cells across a surface facilitated by self-produced surfactin [53]. Interestingly, most isolates were significantly impaired in both swarming and swimming motility (Fig. 4B and C). Swimming motility was observed for the ancestor and evolved isolates after $4 \mathrm{~h}$ (Fig. 4B). However, after $6 \mathrm{~h}$ only the ancestor and Ev6.2 had reached the edge of the petri dish, while the remaining isolates reached at the most half of the swimming distance of the ancestor. Swarming was observed for the ancestor after $4 \mathrm{~h}$ which continued until the expanding colony almost reached the edge of the petri dish after $8 \mathrm{~h}$. In contrast, the evolved isolates showed reduced or a complete lack of swarming throughout the experiment (Fig. 4C). The evolution of motility-impaired isolates in independent populations could indicate that motility is not important for root colonization in the selective environment. Notably, during the EE the 48-well plates were continuously shaking at $90 \mathrm{rpm}$, which may allow the bacteria to get into contact with the root by chance. To test whether motility is important during root colonization under shaking conditions, the ancestor was competed against a $\Delta$ hag mutant, deficient in the production of the flagellin protein, for three successive rounds of root colonization under static or shaking conditions (200 rpm). Under static conditions, the $\Delta$ hag mutant was significantly outcompeted by 
bioRxiv preprint doi: https://doi.org/10.1101/2021.07.09.451762; this version posted July 10, 2021. The copyright holder for this preprint (which was not certified by peer review) is the author/funder, who has granted bioRxiv a license to display the preprint in perpetuity. It is made available under aCC-BY-NC-ND 4.0 International license.

the WT (Fig. S2). In contrast, under shaking conditions, the $\Delta$ hag mutant was able to co-colonize the root to similar levels as the WT. These results demonstrate that motility is important for competition on the root under static conditions but is not required under shaking conditions. Thereby, impaired motility of several of the isolates is not expected to negatively influence the fitness of these isolates in the selective environment.

A
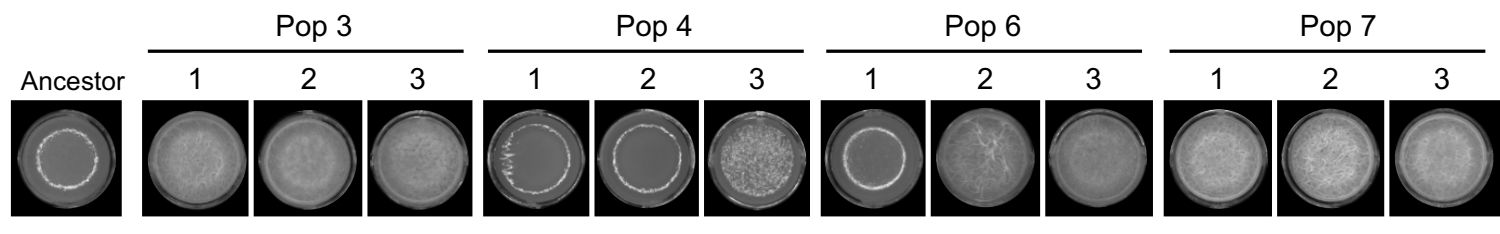

B

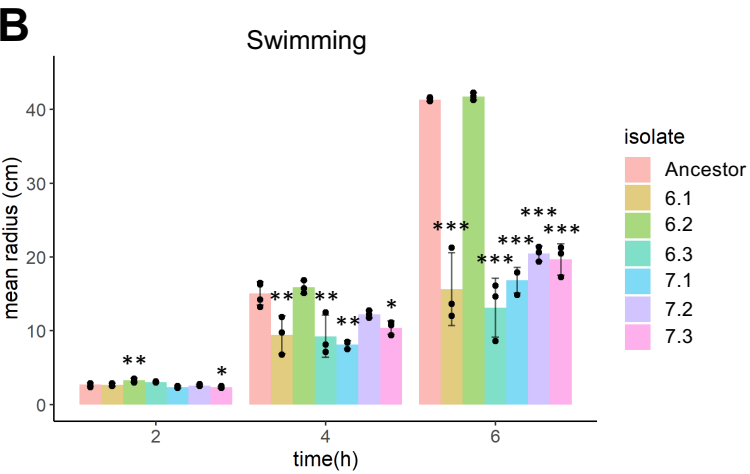

C

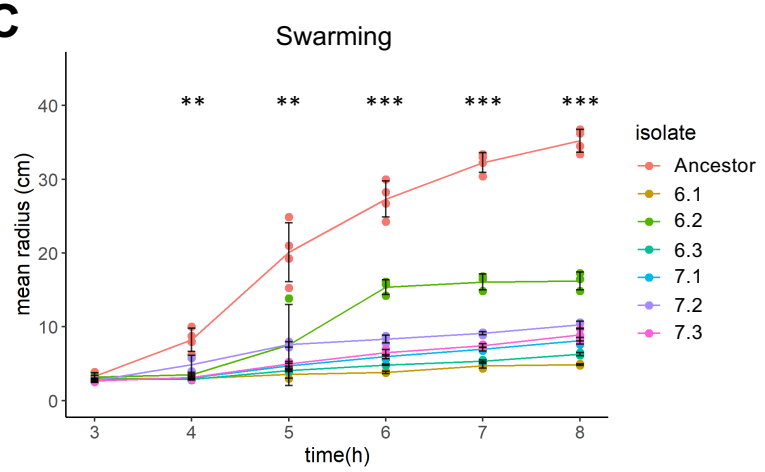

Fig. 4: Evolved isolates from T30 show altered pellicle biofilm formation in response to plant polysaccharides and impaired motility. (A) Ancestor and evolved isolates from transfer 30 were inoculated into LB $+0.5 \%$ xylan at a starting $O D_{600}$ of 0.05 in 24-well plates. Images were acquired after $48 \mathrm{~h}$ incubation at $30^{\circ} \mathrm{C}$ using a stereomicroscope. Each image is representative of four replicates. Evolved isolates were tested for swimming (B) and swarming (C) motility in LB medium supplemented with 0.3 or $0.7 \%$ agar, respectively. (B) Bars represent the mean $(\mathrm{N}=3-4)$ and error bars represent standard deviation. (C) Lines represent the mean $(\mathrm{N}=2-4)$ and error bars the standard deviation. For the motility assays, the following statistical analysis applies: For each time point, an ANOVA was performed on the log10-transformed data followed by a Dunnett's Multiple Comparison test with the ancestor as the control. For swarming motility, the asterisks show the least significance observed for the given time point. At $3 \mathrm{~h}$, only isolate 7.3 was significantly reduced in swarming motility. ${ }^{*}: \mathrm{P}<0.05,{ }^{* *}: \mathrm{P}<0.01,{ }^{* * *}: \mathrm{P}<0.001$.

Evolved isolates show distinct growth profiles in a plant-mimicking environment

The minimal medium (MSNg) used in the EE should render the bacteria dependent on root exudates and dead plant material to survive. A simple way of adapting to this selective environment could be through 
the enhanced utilization of available plant compounds. To test this, we used a modified version of the minimal medium employed during the EE. Instead of $0.05 \%$ glycerol, the MSN medium was supplemented with $0.5 \%$ cellobiose (MSNc). Cellobiose is a disaccharide and a product of partial hydrolysis of cellulose, found in plant cell walls $[19,54]$. In addition, MSNc was supplemented with $0.5 \%$ xylan. The ancestor showed a growth profile typical of bacterial growth under planktonic conditions (Fig. S3). In contrast, several evolved isolates displayed distinct growth profiles, including 3.2, 7.1, 7.2 and 7.3, which showed no decline phase, but instead displayed a prolonged stationary phase. When analyzing the carrying capacity (K), several isolates showed significantly increased carrying capacity (all three isolates from populations 3 and 7), while few isolates showed significantly decreased carrying capacity (Ev4.2, Ev4.3 and Ev6.2). While cellobiose and xylan do not completely represent the plant compounds present in the selective environment, these results suggest that adaptation to the plant root could also be facilitated through the altered utilization of plant compounds.

Evolved isolates harbor mutations in differentiation pathways

To identify the genetic changes that might explain the observed altered phenotypes, the genomes of selected evolved isolates were re-sequenced. The isolates from populations 6 and 7 at T30 were included as these comprised isolates that were differently affected in several traits (Fig. 2-4, Fig. S3). Furthermore, to track molecular evolution over time, the three isolates from population 7 at T12 and T18 were also resequenced. Finally, one isolate from population 1 (Ev1.1) at T30 was included for re-sequencing due to its "Smooth" colony morphology and reduced root colonization (Fig. S4). In the 13 re-sequenced isolates, we observed in total 51 unique mutations (SNPs) of which 37 were non-synonymous (Table S1). All three isolates in population 7 at T30 harbored a mutation in the intergenic region upstream of the $\sin R$ gene encoding a transcriptional repressor of the genes responsible for matrix production $[22,23]$. Interestingly, 
this mutation was also present in the three isolates in population 7 at T18 and in one of the isolates in this population at $\mathrm{T} 12$, suggesting that this mutation arose rather early in the $\mathrm{EE}$ and rose to high frequency or even possibly became fixed in population 7. Two isolates from population 6 harbored a nonsynonymous point mutation in the fliM gene. Additionally, most isolates including Ev1.1 harbored mutations in different genes related to carbon metabolism such as sacC encoding a levanase [55] and gtaB encoding a UTP-glucose-1-phosphate uridylyltransferase that synthesizes a nucleotide sugar precursor essential for the biosynthesis of exopolysaccharides [56] (Table S1).

Adaptation to the root is associated with an evolutionary cost

Adaptation to one environment may result in loss of fitness in different environments [57, 58] representing an evolutionary cost. The three isolates in population 7 at T30 formed Snow-type colonies (Fig. 2) and developed hyper-wrinkled, robust pellicles (Fig. 4A, Fig. S1) indicative of enhanced matrix production. In support of the latter, all three isolates harbored a mutation upstream from $\sin R$ (Table S1), encoding a repressor of matrix gene expression. While we expect these altered phenotypes to confer a fitness advantage during root colonization, i.e. in the selective environment, robust biofilm formation and possibly increased matrix production may represent a disadvantage under other conditions, where biofilm formation is not required for survival or confers a benefit. For example, the EPS and TasA components of the $B$. subtilis biofilm matrix are costly to produce [21]. To investigate whether adaptation to the plant root is accompanied by an evolutionary cost resulting in loss of fitness in another different environment, we competed Ev7.3 against the ancestor during root colonization and in LB + xylan under well-shaking conditions. Following $48 \mathrm{~h}$ of root colonization, CFU quantification revealed that Ev7.3 had outcompeted the ancestor on the root, and statistical analysis confirmed that Ev7.3 had a significantly higher fitness relative to the ancestor (Fig. 5A; for calculation of relative fitness, see Materials and methods). This was 
further supported by CLSM imaging: regardless of the fluorescence labeling combination, Ev7.3 formed robust biofilms on the root evidenced by large aggregates along the root, while the ancestor was scarcely present (Fig. 5C). In contrast, under shaking conditions in LB + xylan, Ev7.3 seemed to suffer a disadvantage compared to the ancestor, although the difference was not significant (Fig. 5B). Similar results were obtained for the permuted fluorescent combination (Fig. S5). To test whether this phenomenon was also true for isolates without the robust pellicle phenotype, we repeated the same experiments with another evolved isolate. Ev6.1 was impaired in motility but not majorly affected in colony morphology or pellicle development (Fig. 2, 4), and only showed a slightly increased individual root colonization (Fig. 3). Nonetheless, during root colonization, Ev6.1 outcompeted the ancestor (Fig. 5A), although the fluorescent images revealed less and smaller aggregates along the root compared to Ev7.3 (Fig. 5C), consistent with the differences in individual root colonization and biofilm formation of the two isolates (Fig. 3, 4A). Under shaking conditions in LB + xylan, Ev6.1 similarly suffered a fitness disadvantage compared to the ancestor (Fig. 5B). These results indicate that adaptation to the root, whether or not associated with the evolution of a robust biofilm phenotype, is accompanied by an evolutionary cost conferring a fitness disadvantage in other environments. 

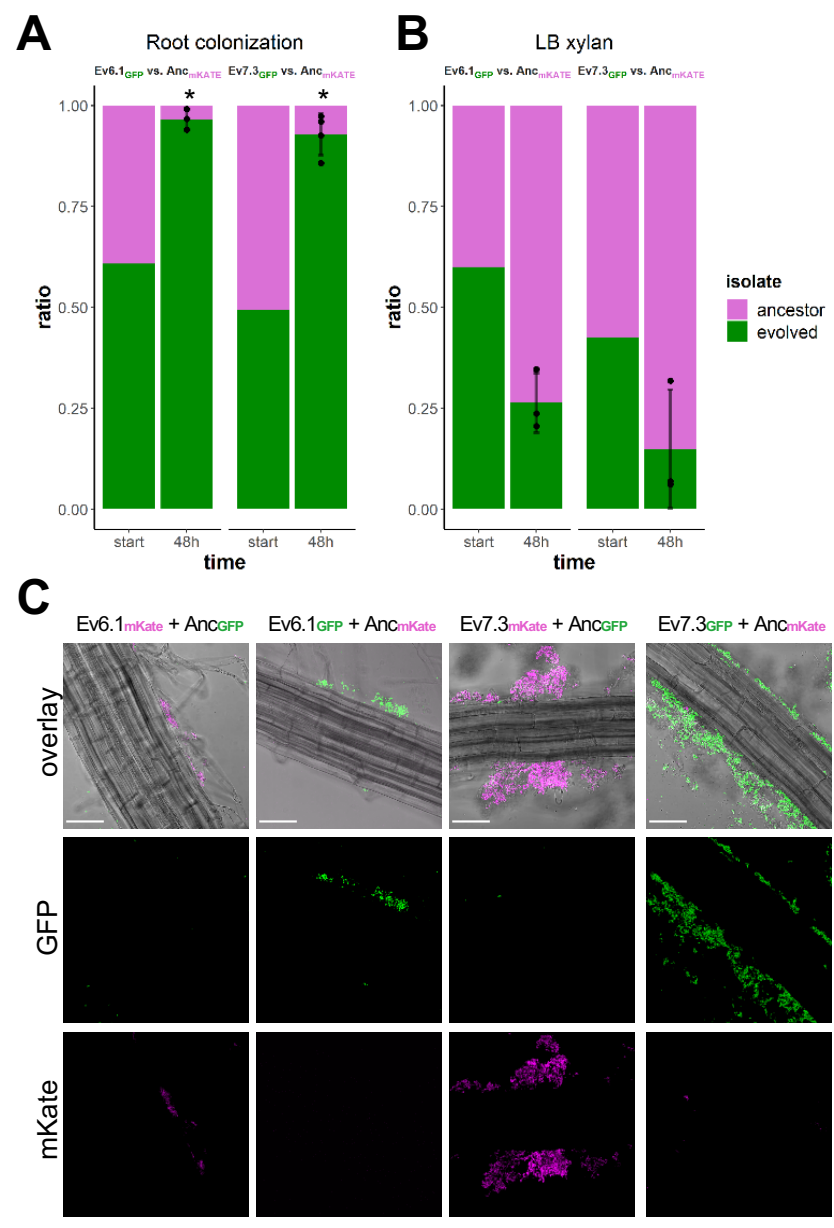

Fig. 5: Two evolved isolates from transfer 30 outcompete the ancestor on the root but suffer a fitness disadvantage under shaking conditions in LB + xylan. A: Pairwise competitions between ancestor (mangenta) and evolved isolates (green) during root colonization (A) and in LB xylan (0.5\%) under shaking conditions (B) for $48 \mathrm{~h}$. In A and B, the bar plots show the starting ratio of the evolved isolate and ancestor in the mix, and the observed ratios after $48 \mathrm{~h}$. Bars represent the mean $(\mathrm{N}=3-4)$, the error bars represent the standard deviation, and the points show the replicates. For statistical analysis, the relative fitness ( $r$ ) of the evolved isolates was calculated by comparing the frequency of the evolved isolate at the beginning and at the end of the competition experiment. The log2-transformed relative fitness values were subjected to a One-sample $t$-test to test whether the mean was significantly different from 0 . *: $\mathrm{P}<0.05$. (C) A. thaliana roots colonized by a 1:1 mix of ancestor and evolved isolates imaged by CLSM. Both fluorescence combinations are shown. The top row shows the overlay of the fluorescence channels and the bright field image. Images are representative of three independent $A$. thaliana seedlings. Color codes are shown at the top. Scale bar is $50 \mu \mathrm{m}$.

An evolved isolate shows increased colonization of $A$. thaliana roots in the presence of a synthetic, soilderived community 
During the $\mathrm{EE}, B$. subtilis was adapted to the plant root alone - in the absence of other microbes. This selective environment is far from its natural habitat in the rhizosphere, where $B$. subtilis encounters other microbial residents. In fact, the ancestor DK1042 is a derivate of the wild strain NCIB 3610, originally isolated from hay infusion $[59,60]$. To this end, we wondered how the pro-longed adaptation of $B$. subtilis to the plant root environment in the absence of other microbial species affected the ability to colonize the root in the presence of such. The ancestor and Ev7.3 were tested for their ability to colonize $A$. thaliana roots in the presence of a synthetic, soil-derived community [38]. This community comprises four bacterial species, Pedobacter sp., Rhodococcus globerulus, Stenotrophomas indicatrix and Chryseobacterium sp. isolated from soil samples that also contained B. subtilis, thereby representing bacterial soil inhabitants. The isolate Ev7.3 was chosen for this test since it was highly adapted to the selective environment, i.e. the isolate displayed significantly increased individual root colonization (Fig. 3) and outcompeted the ancestor during root colonization where it formed a robust biofilm along the root (Fig. 5A, C). To capture any potential difference in the establishment on the root, here defined as root colonization after $48 \mathrm{~h}$, between B. subtilis ancestor and Ev7.3 in the presence of the community, ancestor or Ev7.3 was coinoculated with the community in four different ratios: $0.1: 1,1: 1,10.1$ and 100:1 of $B$. subtilis and community, respectively. When B. subtilis was initially under-represented or highly in excess, i.e. inoculation ratio 0.1:1 and 100:1, respectively, no significant difference was observed in the establishment on the root between B. subtilis ancestor and Ev7.3 within the same inoculation ratio (Fig. 6). In contrast, when co-inoculated with the community in intermediate ratios, i.e. 1:1 and 10:1, isolate Ev7.3 showed significantly enhanced establishment on the root compared to the ancestor. Since Ev7.3 displayed increased carrying capacity in MSNc + xylan in monoculture compared to the ancestor (Suppl. Fig. 3), we wondered whether the enhanced establishment on the root by Ev7.3 in the presence of the community could be partly attributed to improved utilization of plant compounds. Indeed, growth profiles in MSNc + xylan of the ancestor or Ev7.3 in co-culture with the community revealed that Ev7.3 displayed a 
bioRxiv preprint doi: https://doi.org/10.1101/2021.07.09.451762; this version posted July 10, 2021. The copyright holder for this preprint (which was not certified by peer review) is the author/funder, who has granted bioRxiv a license to display the preprint in perpetuity. It is made available under aCC-BY-NC-ND 4.0 International license.

significantly increased carrying capacity at inoculation ratio 1:1, 10:1 and 100:1 compared to the ancestor

(Fig. S6). Finally, in vitro confrontation assays on LB agar (1.5\%) showed no major difference in the inhibition of the community members by Ev7.3 compared to the ancestor (Fig. S7). Taken together, these results show that even though $B$. subtilis was adapted to the plant root alone, isolate Ev7.3 displayed increased root colonization also in the presence of a semi-synthetic, soil-derived community under certain inoculation ratios, possibly mediated by robust biofilm formation on the root and enhanced utilization of plant compounds.

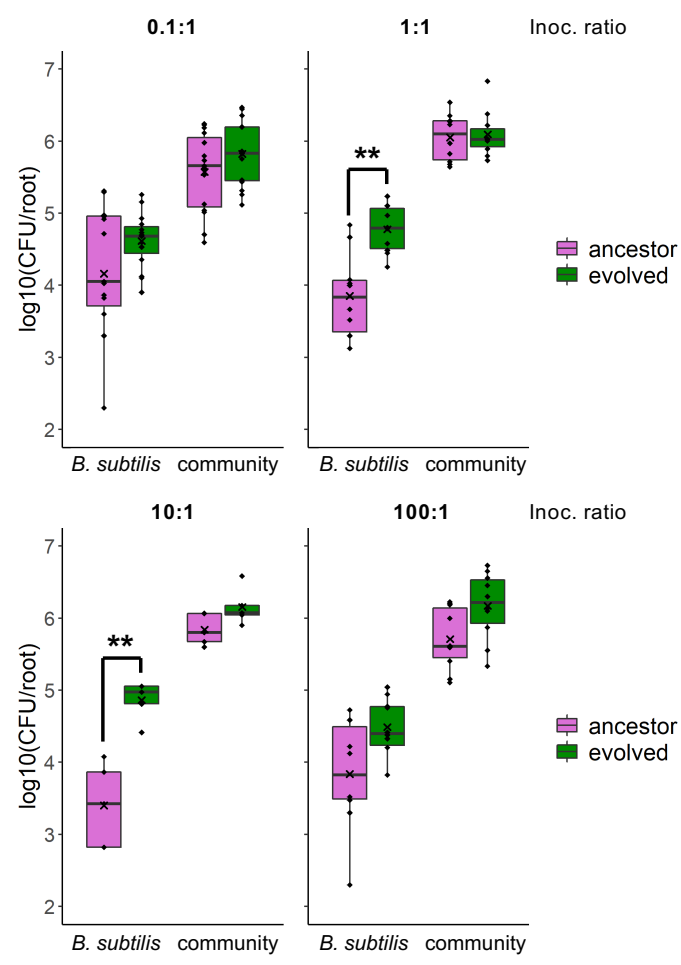

Fig. 6: Root colonization by B. subtilis ancestor and isolate Ev7.3 in the presence of a semi-synthetic, soil-derived community. The ancestor and evolved isolate, Ev7.3, were tested for the ability to colonize the root in the presence of a semi-synthetic, soilderived bacterial community. B. subtilis ancestor or Ev7.3 and the community were co-inoculated onto $A$. thaliana roots in four different ratios: $0.1: 1,1: 1,10: 1$ and 100:1 of $B$. subtilis and community, respectively. Root colonization after $48 \mathrm{~h}$ was quantified as $\log 10$-transformed productivity (CFU/root). Each plot shows the resulting root colonization at the given inoculation ratio of $B$. subtilis (left) and the co-inoculated community (right). Magenta: Ancestor and the corresponding community co-inoculated with the ancestor. Green: Ev7.3 and the community co-inoculated with Ev7.3. The cross represents the mean (N=5-15). Within each 
inoculation ratio, statistical significance between $B$. subtilis ancestor and Ev7.3, and between the communities co-inoculated with the ancestor or with Ev7.3 was tested with a Two-sample $t$-test (Welch's Two-sample $t$-test when unequal variance). ${ }^{* *}: \mathrm{P}<0.01$.

\section{Discussion}

Several studies have reported experimental evolution as a powerful tool to explore how bacteria adapt to ecologically relevant environments. A recent study investigated the adaptive response of the PGPR Pseudomonas protegens to the $A$. thaliana rhizosphere in a sand system, which revealed mutations in genes encoding global regulators and genes related to motility and cell surface structure across independent populations [61] and during such adaptation, the initially plant-antagonistic $P$. protegens bacterium evolved into mutualists [44]. Furthermore, Lin et al. (2020) observed that adaptation of Bacillus thuringiensis to $A$. thaliana roots under hydroponic conditions led to the evolution of multicellular aggregating phenotypes, which, surprisingly, in certain lineages were accompanied by enhanced virulence against the Galleria mellonella larvae. Here, we demonstrate that $B$. subtilis rapidly adapts to $A$. thaliana roots under hydroponic conditions, as observed by the presence of evolved isolates at transfer 12 displaying improved root colonization and harboring several genetic changes compared to the ancestor. Further phenotypic characterization of the evolved isolates from the final transfer revealed that most isolates across independent populations developed more robust biofilms in response to the plant polysaccharide xylan compared to the ancestor. Except for isolate Ev3.3, the robust biofilm formers tended to be increased in individual root colonization, indicating that robust biofilm formation is associated with adaptation to the plant root. Motility represents an important trait for many bacteria as it allows them to explore the environment for nutrients and escape unfavorable conditions. Of relevance to the adaptation of $B$. subtilis to plant roots, motility has been shown to be important for root colonization of different plant species under different conditions. For example, a $B$. subtilis $\Delta$ hag mutant 
was shown to be delayed or reduced in A. thaliana root colonization under hydroponic condititions as well as in tomato root colonization under vermiculites pot conditions $[15,16]$. Yet, we found that five out of six isolates from two independent populations were impaired in both swimming and swarming motility, indicating that motility is not important for root colonization in the selective environment of the $\mathrm{EE}$, i.e. under hydroponic, shaking conditions. Indeed, this was verified in a competition experiment between a non-motile $\Delta$ hag mutant and the WT, revealing that motility is not required for root colonization under shaking conditions. In contrast to our observations, Li et al. (2021) observed several evolved isolates of $P$. protegens improved in swimming motility following adaptation to the $A$. thaliana rhizosphere in a sand system [61], supporting that in sand (and thus possibly also in soil), motility is indeed important for root colonization and is therefore selected for.

In B. subtilis, motility and biofilm formation are incompatible processes: $B$. subtilis can exist as single, motile cells or in chains of sessile, matrix-producing cells which is regulated by an epigenetic switch involving $\operatorname{Sin} R[62,63]$. The enhanced biofilm formation and impaired motility of isolates from populations 6 and 7 (Ev6.3, Ev7.1, Ev7.2 and Ev7.3) could thereby indicate a possible biofilm-motility trade-off. An inverse evolutionary trade-off between biofilm formation and motility was observed when the opportunistic pathogen Pseudomonas areginosa was subjected to repeated rounds of swarming which lead to the evolution of hyper-swarmers that were impaired in biofilm formation [64]. Considering that the ability to form robust biofilm in vitro was shown to positively correlate with root colonization in $B$. subtilis [18], and the demonstration that motility is not important for root colonization under shaking conditions, a possible biofilm-motility trade-off could provide $B$. subtilis with enhanced fitness during root colonization in the selective environment. Indeed, isolate Ev7.3, which developed hyper-robust biofilms in LB + xylan and was impaired in motility, significantly outcompeted the ancestor during root colonization. 
Re-sequencing of selected evolved isolates revealed that Ev7.1, Ev7.2 and Ev7.3 (from transfer 30) all harbored a mutation in the intergenic region upstream of the $\sin R$ gene, encoding a transcriptional repressor of matrix genes $[22,23]$. This SNP was located two base pairs upstream from the start codon [65] and could thereby potentially affect translation efficiency from the mRNA transcript. Reduced levels of SinR caused by the intergenic mutation could result in increased expression of matrix genes. This is supported by Richter et al. (2018) who demonstrated that a $\Delta \sin R$ mutant shows increased matrix gene expression, and by Subramaniam et al. (2013) reporting that SinR translation and therefore protin level affects matrix gene expression. Potential increased matrix production caused by this mutation could contribute to the Snow-type colony morphology, as this colony morphology was exclusively observed for isolates harbouring this mutation. Furthermore, in accordance to the robust biofilm formation and increased root colonization observed for Ev7.1, Ev7.2 and Ev7.3, a $\Delta \sin R$ mutant was shown to form a hyper-robust biofilm in biofilm-inducing medium as well as on tomato roots [18], supporting the possible relevance of this mutation for the observed phenotypes of these isolates. These three isolates did not harbor mutations in motility-related genes. However, besides a possible effect of reduced SinR levels on the epigenetic switch [63] that could lock the cells in a sessile, matrix-producing stage, a potential reduction in SinR levels leading to overexpression of the eps operon could possibly reduce motility due to the EpsE clutch [68]. Such mutation and the observed corresponding phenotypes could be responsible for the biofilm-motility trade-off, and be an example of antagonistic pleiotropy [58] in which the same mutation is beneficial in one environment, i.e. during root colonization under shaking conditions, but disadvantageous in another, i.e. where motility is required for survival. In addition, this mutation affecting a biofilm regulator could possibly explain why Ev7.1, Ev7.2 and Ev7.3 show improved biofilm formation also in the absence of xylan.

Isolate Ev6.1 and Ev6.3 harboured a non-synonymous point mutation in the fliM gene, which was not present in Ev6.2. This gene encodes a flagellar motor switch protein, part of the basal body C-ring 
controlling the direction of flagella rotation [69]. Interestingly, Ev6.1 and Ev6.3 were impaired in both forms of motility, while Ev6.2 showed similar swimming as the ancestor and was less affected in swarming. We speculate that the R326I substitution affects the function of FliM and consequently the flagellar machinery, resulting in hampered motility in these two isolates. Since we showed that motility was not important for root colonization under shaking conditions, a mutation hampering motility could provide the bacterium a fitness advantage during the adaptation to $A$. thaliana roots due to the reduced cost of this apparently redundant trait. However, we do not expect the mutation in fliM to result in reduced cost; it merely changes an amino acid in a protein part of the flagellar machinery. Other mutations in the population 6 isolates must explain the robust biofilm formation by Ev6.2 and Ev6.3 and the fitness advantage of Ev6.1 over the ancestor during root colonization. For example, isolate Ev6.1 and Ev6.3 harbor a mutation in kinA encoding a two-component sensor kinase which once activated initiates the phosphorelay leading to phosphorylation of the master regulator Spo0A [24].

The isolate from population 1 at transfer 30 (Ev1.1) harbored a frameshift mutation in the rsiX locus, encoding an anti-sigma factor controlling the activity of SigX [70]. Inconsistent with the smooth morphology and reduced root colonization of this isolate, an $\Delta r$ riX mutant was shown to have increased eps expression [47]. However, Ev1.1 additionally harbored several mutations in gtaB encoding a UTPglucose-1-phosphate uridylyltransferase involved in the biosynthesis of a nucleotide sugar precursor for EPS [56]. A study conducted by Reverdy et al. (2018) showed that acetylation of GtaB is important for biofilm formation and that a gtaB mutant was reduced in pellicle formation. We therefore speculate, that Ev1.1 may have first gained the mutation in rsiX and was selected for due to increased eps expression, while later on the increased matrix production was reverted by the mutations in gtaB, resulting in a nonfunctional protein, which was selected for due to reduced cost. In accordance with the mutations observed in this study, a non-synonymous mutation in $\sin R$ in two isolates forming Snow-type colonies and increased in root colonization as well as several mutations in gtaB in two isolates with a Smooth 
colony morphology were observed in our recent study on diversification of $B$. subtilis during adaptation to $A$. thaliana roots [11].

While the phenotypic and genetic changes of isolates Ev6.1 and Ev7.3 conferred a fitness advantage in competition with the ancestor during root colonization, adaptation to one certain environment may be accompanied by a loss of fitness in other environments [58]. This has been demonstrated for Escherichia coli which following adaptation to low temperature showed reduced fitness at high temperature [57]. In the example of the evolution of hyperswarmers of $P$. areginosa, the hyperswarmer clones outcompeted the ancestor in swarming, but lost in biofilm competitions [64]. In this study, we demonstrate that adaptation of $B$. subtilis to $A$. thaliana roots is accompanied by an evolutionary cost. When Ev6.1 and Ev7.3 each were competed against the ancestor in LB + xylan under shaking conditions, i.e. an environment where plant compounds are present but biofilm formation is not required for survival, both evolved isolates suffered a fitness disadvantage. The observation that two evolved isolates, from independent populations and with different phenotypes and genetic changes, both suffered a fitness disadvantage in a different environment might suggest the generality of such an evolutionary cost during adaptation to $A$. thaliana roots.

In our EE approach, B. subtilis was adapted to plant roots in the absence of other microbes. In the rhizosphere environment under natural conditions, $B$. subtilis is far from being the sole microbial inhabitant but it engages in cooperative and competitive interactions with other members of the rhizosphere microbiome $[12,32]$. We tested whether the evolved isolate, Ev7.3, displaying increased root colonization in the selective environment relative to the ancestor, would also show improved establishment on the root under more ecologically complex conditions. We found that in the precence of a synthetic soil-derived community, Ev7.3 displayed enhanced establishment on the root compared to the ancestor in two out of four inoculation ratios. This enhanced establishment on the root by Ev7.3 is not expected to be caused by altered antagonistic activities towards the community members. First, no major 
changes in the inhibition of the community members were observed in confrontation colony assays.

Secondly, an increased number of Ev7.3 cells on the root did not cause a reduction in the co-colonizing community. Finally, Ev7.3 did not harbor mutations in genes directly related to secondary metabolite production. Instead, enhanced establishment on the root by Ev7.3 in the presence of the community is possibly enabled by robust biofilm formation facilitating stronger attachment to the root and enhanced utilization of plant compounds. Interestingly, a study by Molina-Santiago et al. showed that compared to a $\Delta$ matrix mutant, co-inoculation of $B$. subtilis WT with Pseudomonas chlororaphis on melon leaves enabled co-localization of the two species as well as closer attachment of $B$. subtilis to the leave surface [72]. The robust biofilm formed on the root by Ev7.3 possibly facilitated by increased matrix production may thereby not exclude the community members on the root but could rather allow them to incorporate into the matrix. This could also explain why the enhanced establishment of B. subtilis Ev7.3 on the root did not cause a reduction in the number of community cells attached to the root. Alternatively, the community may not be majorly affected by any difference in establishment on the root between the ancestor and Ev7.3 due to the low abundance of $B$. subtilis relative to the community. Further work is needed to elucidate the interactions between $B$. subtilis and this synthetic community during root colonization. In summary, these findings suggest that even though B. subtilis was evolved on A. thaliana in the absence of other microbes, it became highly adapted to the root environment enabling better establishment on the root also when the ecological complexity increases. How genetic adaptation to the plant root in the absence of other microbial species differs from adaptation to plant root environments with varying levels of ecological complexity is the scope of future studies.

The formation of root-associated biofilms is important for the biocontrol efficacy of $B$. subtilis [18]. From an applied perspective, experimental evolution of $B$. subtilis on plant roots represents a novel approach for developing strains with enhanced root attachment capacities for agricultural use. However, a biofilmmotility tradeoff as observed here may be undesirable when developing biocontrol agents due to the 
growing evidence of motility as an important trait for bacterial root colonization in soil systems $[16,61]$.

The phenotypes associated with adaptation of $B$. subtilis to $A$. thaliana roots presented here as well as the accompanying evolutionary cost and the increased root colonization even in the presence of resident soil bacteria highlight the importance of considering the selective environment if evolving PGPR for biocontrol purposes.

\section{Acknowledgements}

We thank Carlos N. Lozano-Andrade for the four bacterial species constituting the synthetic community. The work was supported by a DTU Bioengineering start-up fund to ÁTK. Fundings from Novo Nordisk Foundation for the infrastructure "Imaging microbial language in biocontrol (IMLiB)" (NNFOC0055625) and within the INTERACT project of the Collaborative Crop Resiliency Program (NNF19SA0059360) are acknowledged.

Authors' contributions: M.N.C. and Á.T.K. conceived the project; M.N.C. and C.B. performed experiments; M.N.C. and M.L.S analyzed the data; G.M. performed the re-sequencing and the belonging data analysis; M.N.C. and Á.T.K. wrote the manuscript with feedback from all authors.

\section{Conflict of interests}

The authors declare that there is no conflict of interests in relation to the work described. 


\section{References}

1. Egamberdieva D, Kamilova F, Validov S, Gafurova L, Kucharova Z, Lugtenberg B. High incidence of plant growth-stimulating bacteria associated with the rhizosphere of wheat grown on salinated soil in Uzbekistan. Environ Microbiol 2008; 10: 1-9.

2. Mendes R, Kruijt M, De Bruijn I, Dekkers E, Van Der Voort M, Schneider JHM, et al. Deciphering the rhizosphere microbiome for disease-suppressive bacteria. Science (80- ) 2011; 332: 10971100.

3. Berendsen RL, Vismans G, Yu K, Song Y, De Jonge R, Burgman WP, et al. Disease-induced assemblage of a plant-beneficial bacterial consortium. ISME J 2018; 12: 1496-1507.

4. Rudrappa T, Czymmek KJ, Paré PW, Bais HP. Root-secreted malic acid recruits beneficial soil bacteria. Plant Physiol 2008; 148: 1547-1556.

5. Berendsen RL, Pieterse CMJ, Bakker PAHM. The rhizosphere microbiome and plant health. Trends Plant Sci 2012; 17: 478-486.

6. Trivedi P, Leach JE, Tringe SG, Sa T, Singh BK. Plant-microbiome interactions: from community assembly to plant health. Nat Rev Microbiol 2020; 18: 607-621.

7. Cazorla FM, Romero D, Pérez-García A, Lugtenberg BJJ, Vicente A De, Bloemberg G. Isolation and characterization of antagonistic Bacillus subtilis strains from the avocado rhizoplane displaying biocontrol activity. J Appl Microbiol 2007; 103: 1950-1959.

8. Huang Y, Wu Z, He Y, Ye BC, Li C. Rhizospheric Bacillus subtilis exhibits biocontrol effect against Rhizoctonia solani in Pepper (Capsicum annuum). Biomed Res Int 2017; 2017.

9. Fall R, Kinsinger RF, Wheeler KA. A simple method to isolate biofilm-forming Bacillus subtilis and related species from plant roots. Syst Appl Microbiol 2004; 27: 372-379.

10. Pandey A, Palni LMS. Bacillus species: The dominant bacteria of the rhizosphere of established tea bushes. Microbiol Res 1997; 152: 359-365.

11. Blake C, Christensen MN, Kovács ÁT. Molecular aspects of plant growth promotion and protection by Bacillus subtilis. Mol Plant Microbe Interact 2021; 34: 15-25.

12. Kiesewalter HT, Lozano-Andrade CN, Wibowo M, Strube ML, Maróti G, Snyder D, et al. Genomic and chemical diversity of Bacillus subtilis secondary metabolites against plant pathogenic fungi. mSystems 2021; 6: e00770-20.

13. Ongena M, Jacques P. Bacillus lipopeptides: versatile weapons for plant disease biocontrol. Trends Microbiol 2008; 16: 115-125.

14. Fira D, Dimkić I, Berić T, Lozo J, Stanković S. Biological control of plant pathogens by Bacillus species. J Biotechnol 2018; 285: 44-55.

15. Allard-Massicotte R, Tessier L, Lécuyer F, Lakshmanan V, Lucier JF, Garneau D, et al. Bacillus subtilis early colonization of Arabidopsis thaliana roots involves multiple chemotaxis receptors. MBio 2016; 7: e01664-16.

16. Tian T, Sun B, Shi H, Gao T, He Y, Li Y, et al. Sucrose triggers a novel signaling cascade promoting Bacillus subtilis rhizosphere colonization. ISME J 2021. 
17. Bais HP, Fall R, Vivanco JM. Biocontrol of Bacillus subtilis against Infection of Arabidopsis Roots by Pseudomonas syringae Is Facilitated by Biofilm Formation and Surfactin Production. Plant Physiol 2004; 134: 307-319.

18. Chen Y, Yan F, Chai Y, Liu H, Kolter R, Losick R, et al. Biocontrol of tomato wilt disease by Bacillus subtilis isolates from natural environments depends on conserved genes mediating biofilm formation. Environ Microbiol 2013; 15: 848-864.

19. Beauregard PB, Chai Y, Vlamakis H, Losick R, Kolter R. Bacillus subtilis biofilm induction by plant polysaccharides. Proceedings of the National Academy of Sciences of the United States of America. 2013. pp 1621-1630.

20. Branda SS, Chu F, Kearns DB, Losick R, Kolter R. A major protein component of the Bacillus subtilis biofilm matrix. Mol Microbiol 2006; 59: 1229-1238.

21. Dragoš A, Kiesewalter H, Martin M, Hsu CY, Hartmann R, Wechsler T, et al. Division of labor during biofilm matrix production. Curr Biol 2018; 28: 1903-1913.e5.

22. Kearns DB, Chu F, Branda SS, Kolter R, Losick R. A master regulator for biofilm formation by Bacillus subtilis. Mol Microbiol 2005; 55: 739-749.

23. Chu F, Kearns DB, Branda SS, Kolter R, Losick R. Targets of the master regulator of biofilm formation in Bacillus subtilis. Mol Microbiol 2006; 59: 1216-1228.

24. Jiang $\mathrm{M}$, Shao W, Perego $\mathrm{M}$, Hoch JA. Multiple histidine kinases regulate entry into stationary phase and sporulation in Bacillus subtilis. Mol Microbiol 2000; 38: 535-542.

25. Fujita M, González-Pastor JE, Losick R. High- and low-threshold genes in the Spo0A regulon of Bacillus subtilis. J Bacteriol 2005; 187: 1357-1368.

26. Bai U, Mandic-Mulec I, Smith I. Sinl modulates the activity of SinR, a developmental switch protein of Bacillus subtilis, by protein-protein interaction. Genes Dev 1993; 7: 139-148.

27. Chen Y, Cao S, Chai Y, Clardy J, Kolter R, Guo JH, et al. A Bacillus subtilis sensor kinase involved in triggering biofilm formation on the roots of tomato plants. Mol Microbiol 2012; 85: 418-430.

28. Asaka O, Shoda M. Biocontrol of Rhizoctonia solani damping-off of tomato with Bacillus subtilis RB14. Appl Environ Microbiol 1996; 62: 4081-4085.

29. Lugtenberg B, Kamilova F. Plant-growth-promoting rhizobacteria. Annu Rev Microbiol 2009; 63: 541-556.

30. Köhl J, Kolnaar R, Ravensberg WJ. Mode of action of microbial biological control agents against plant diseases: Relevance beyond efficacy. Front Plant Sci 2019; 10: 1-19.

31. Akram W, Anjum T, Ali B. Searching ISR determinant/s from Bacillus subtilis IAGS174 against Fusarium wilt of tomato. BioControl 2015; 60: 271-280.

32. Hassani MA, Durán $\mathrm{P}$, Hacquard S. Microbial interactions within the plant holobiont. Microbiome 2018; $6: 58$.

33. Lenski RE. What is adaptation by natural selection? Perspectives of an experimental microbiologist. PLoS Genet 2017; 13: 1-12.

34. Kawecki TJ, Lenski RE, Ebert D, Hollis B, Olivieri I, Whitlock MC. Experimental evolution. Trends 
Ecol Evol 2012; 27: 547-560.

35. Dragoš A, Lakshmanan N, Martin M, Horváth B, Maróti G, García CF, et al. Evolution of exploitative interactions during diversification in Bacillus subtilis biofilms. FEMS Microbiol Ecol 2018; 93: fix155.

36. Poltak SR, Cooper VS. Ecological succession in long-term experimentally evolved biofilms produces synergistic communities. ISME J 2011; 5: 369-378.

37. Konkol MA, Blair KM, Kearns DB. Plasmid-encoded Coml inhibits competence in the ancestral 3610 strain of Bacillus subtilis. J Bacteriol 2013; 195: 4085-4093.

38. Lozano-Andrade CN, Strube ML, Kovács ÁT. Complete genome sequences of four new isolates constituting a stable soil-derived synthetic bacterial community. Submitted 2021.

39. Casadaban MJ, Chou J, Cohen SN. In vitro gene fusions that join an enzymatically active Bgalactosidase segment to amino-terminal fragments of exogenous proteins: Escherichia coli plasmid vectors for the detection and cloning of translational initiation signals. J Bacteriol 1980; 143: 971-980.

40. Nordgaard M, Mortensen RMR, Kirk NK, Gallegos-Monterrosa R, Kovács ÁT. Deletion of Rap-Phr systems in Bacillus subtilis influences in vitro biofilm formation and plant root colonization. Microbiologyopen 2021; 10: 1-15.

41. Gallegos-Monterrosa R, Mhatre E, Kovács ÁT. Specific Bacillus subtilis 168 variants form biofilms on nutrient-rich medium. Microbiol (United Kingdom) 2016; 162: 1922-1932.

42. Jousset A, Rochat L, Péchy-Tarr M, Keel C, Scheu S, Bonkowski M. Predators promote defence of rhizosphere bacterial populations by selective feeding on non-toxic cheaters. ISME J 2009; 3: 666-674.

43. Ross-Gillespie A, Gardner A, West SA, Griffin AS. Frequency dependence and cooperation: Theory and a test with bacteria. Am Nat 2007; 170: 331-342.

44. Li E, de Jonge R, Chen L, Friman V, Bakker P, Pieterse C, et al. Rapid evolution of bacterial mutualism in the plant rhizosphere. Nat Commun 2021; 12: 3829.

45. Sprouffske K, Wagner A. Growthcurver: An R package for obtaining interpretable metrics from microbial growth curves. BMC Bioinformatics 2016; 17: 17-20.

46. Dragoš A, Martin M, Garcia CF, Kricks L, Pausch P, Heimerl T, et al. Collapse of genetic division of labour and evolution of autonomy in pellicle biofilms. Nat Microbiol 2018; 3: 1451-1460.

47. Martin M, Dragoš A, Otto SB, Schäfer D, Brix S, Maróti G, et al. Cheaters shape the evolution of phenotypic heterogeneity in Bacillus subtilis biofilms. ISME J 2020; 14: 2302-2312.

48. Thérien M, Kiesewalter HT, Auria E, Charron-Lamoureux V, Wibowo M, Maróti G, et al. Surfactin production is not essential for pellicle and root-associated biofilm development of Bacillus subtilis. Biofilm 2020; 2: 100021.

49. Gallegos-Monterrosa R, Christensen MN, Barchewitz T, Koppenhöfer S, Priyadarshini B, Bálint B, et al. Impact of Rap-Phr system abundance on adaptation of Bacillus subtilis. Commun Biol 2021; 4: 1-12. 
50. Lin Y, Alstrup M, Pang JKY, Maróti G, Er-Rafik M, Tourasse N, et al. Adaptation of Bacillus thuringiensis to plant colonisation affects differentiation and toxicity. bioRxiv 2020; 1130-1134.

51. Branda SS, González-Pastor JE, Ben-Yehuda S, Losick R, Kolter R. Fruiting body formation by Bacillus subtilis. Proc Natl Acad Sci U S A 2001; 98: 11621-11626.

52. Liepman AH, Wightman R, Geshi N, Turner SR, Scheller HV. Arabidopsis - A powerful model system for plant cell wall research. Plant J 2010; 61: 1107-1121.

53. Kearns DB. A field guide to bacterial swarming motility. Nat Rev Microbiol 2010; 8: 634-644.

54. Endler A, Persson S. Cellulose synthases and synthesis in Arabidopsis. Mol Plant 2011; 4: 199211.

55. Martin I, Débarbouillé M, Ferrari E, Klier A, Rapoport G. Characterization of the levanase gene of Bacillus subtilis which shows homology to yeast invertase. Mol Gen Genet 1987; 208: 177-184.

56. Varon D, Boylan SA, Okamoto K, Price CW. Bacillus subtilis gtaB Encodes UDP-Glucose Pyrophosphorylase and Is Controlled by Stationary-Phase Transcription Factor $\sigma \mathrm{B}$. J Bacteriol 1993; 175: 3964-3971.

57. Bennett AF, Lenski RE. An experimental test of evolutionary trade-offs during temperature adaptation. Proc Natl Acad Sci U S A 2007; 104: 8649-8654.

58. Elena SF, Lenski RE. Evolution experiments with microorganisms: The dynamics and genetic bases of adaptation. Nat Rev Genet 2003; 4: 457-469.

59. Zeigler DR, Prágai Z, Rodriguez S, Chevreux B, Muffler A, Albert T, et al. The Origins of 168, W23, and Other Bacillus subtilis Legacy Strains. J Bacteriol 2008; 190: 6983-6995.

60. Cohn HJ. The identity of Bacillus subtilis. J Infect Dis 1930; 46: 341-350.

61. Li E, Zhang $\mathrm{H}$, Jiang $\mathrm{H}$, Pieterse $\mathrm{CMJ}$, Jousset $\mathrm{A}$, Bakker PAHM. Experimental-evolution-driven identification of Arabidopsis rhizosphere competence genes in Pseudomonas protegens. MBio 2021; 12: e00927-21.

62. Vlamakis H, Aguilar C, Losick R, Kolter R. Control of cell fate by the formation of an architecturally complex bacterial community. Genes Dev 2008; 22: 945-953.

63. Chai Y, Kolter R, Losick R. An epigenetic switch governing daughter cell separation in Bacillus subtilis. Genes Dev 2010; 24: 754-765.

64. van Ditmarsch D, Boyle KE, Sakhtah H, Oyler JE, Nadell CD, Déziel É, et al. Convergent evolution of hyperswarming leads to impaired biofilm formation in pathogenic bacteria. Cell Rep 2013; 4: 697-708.

65. Agarwala R, Barrett T, Beck J, Benson DA, Bollin C, Bolton E, et al. Database resources of the National Center for Biotechnology Information. Nucleic Acids Res 2018; 46: D8-D13.

66. Richter A, Hölscher T, Pausch P, Sehrt T, Brockhaus F, Bange G, et al. Hampered motility promotes the evolution of wrinkly phenotype in Bacillus subtilis. BMC Evol Biol 2018; 18: 155.

67. Subramaniam AR, Deloughery A, Bradshaw N, Chen Y, O'Shea E, Losick R, et al. A serine sensor for multicellularity in a bacterium. Elife 2013; 2: e01501. 
bioRxiv preprint doi: https://doi.org/10.1101/2021.07.09.451762; this version posted July 10, 2021. The copyright holder for this preprint (which

was not certified by peer review) is the author/funder, who has granted bioRxiv a license to display the preprint in perpetuity. It is made available under aCC-BY-NC-ND 4.0 International license.

68. Blair KM, Turner L, Winkelman JT, Berg HC, Kearns DB. A molecular clutch disables flagella in the Bacillus subtilis biofilm. Science 2008; 320: 1636-1638.

69. Guttenplan SB, Shaw S, Kearns DB. The cell biology of peritrichous flagella in Bacillus subtilis. Mol Microbiol 2013; 87: 211-229.

70. Zhu B, Stülke J. SubtiWiki in 2018: From genes and proteins to functional network annotation of the model organism Bacillus subtilis. Nucleic Acids Res 2018; 46: D743-D748.

71. Reverdy A, Chen Y, Hunter E, Gozzi K, Chai Y. Protein lysine acetylation plays a regulatory role in Bacillus subtilis multicellularity. PLoS One 2018; 13: e0204687.

72. Molina-Santiago C, Pearson JR, Navarro Y, Berlanga-Clavero MV, Caraballo-Rodriguez AM, Petras $D$, et al. The extracellular matrix protects Bacillus subtilis colonies from Pseudomonas invasion and modulates plant co-colonization. Nat Commun 2019; 10: 1919. 